\title{
Drosophila BRUCE inhibits apoptosis through non-lysine ubiquitination of the IAP-antagonist REAPER
}

\author{
C Domingues ${ }^{1}$ and HD Ryoo, 1
}

Active caspases execute apoptosis to eliminate superfluous or harmful cells in animals. In Drosophila, living cells prevent uncontrolled caspase activation through an inhibitor of apoptosis protein (IAP) family member, dIAP1, and apoptosis is preceded by the expression of IAP-antagonists, such as Reaper, Hid and Grim. Strong genetic modifiers of this pathway include another IAP family gene encoding an E2 ubiquitin conjugating enzyme domain, $d B r u c e$. Although the genetic effects of $d B r u c e$ mutants are well documented, molecular targets of its encoded protein have remained elusive. Here, we report that dBruce targets Reaper for ubiquitination through an unconventional mechanism. Specifically, we show that dBruce physically interacts with Reaper, dependent upon Reaper's IAP-binding (IBM) and GH3 motifs. Consistently, Reaper levels were elevated in a dBruce $-I-$ background. Unexpectedly, we found that $d B r u c e$ also affects the levels of a mutant form of Reaper without any internal lysine residues, which normally serve as conventional ubiquitin acceptor sites. Furthermore, we were able to biochemically detect ubiquitin conjugation on lysine-deficient Reaper proteins, and knockdown of $d B$ ruce significantly reduced the extent of this ubiquitination. Our results indicate that $d B$ ruce inhibits apoptosis by promoting IAP-antagonist ubiquitination on unconventional acceptor sites.

Cell Death and Differentiation (2012) 19, 470-477; doi:10.1038/cdd.2011.116; published online 2 September 2011

Metazoans use a specific form of cell death known as apoptosis to eliminate damaged and potentially dangerous cells. ${ }^{1}$ Indicative of its important role in tissue homeostasis, defective apoptosis is associated with a wide variety of diseases that includes cancer. ${ }^{2}$

Developmental programming or damage response pathways can trigger apoptosis by inducing pro-apoptotic gene expression. In Drosophila, four specific genes grim, reaper, sickle and hid are transcriptionally induced before apoptosis, and are required for most apoptosis that occurs in this organism. ${ }^{3,4}$ The encoded proteins are now widely referred to as IAP-antagonists, as they directly inhibit Drosophila IAP1 (dIAP1), a member of the inhibitor of apoptosis protein (IAP) family that contains signature Baculovirus IAP repeat (BIR) domains. ${ }^{5}$ Consistently, gain- or loss-of-function alleles of dIAP1 have been isolated from independent genetic screens as strong modifiers of IAP-antagonist-induced cell death. ${ }^{6,7}$ Once IAP-antagonists bind, dIAP1 itself undergoes ubiquitinmediated degradation. ${ }^{8-11}$ In living cells, dIAP1 inhibits proapoptotic proteins, such as Dronc (initiator caspase), drICE and DCP-1 (effector caspases), as well as IAP-antagonists by tagging these targets with ubiquitin or ubiquitin-like proteins. ${ }^{12-16}$ Similarly in humans, IAP family members act as strong suppressors of apoptosis and a number of IAPantagonists help sensitize these cells to various death stimuli. ${ }^{17-19}$ This pathway has drawn particular interest in recent years as abnormally high IAP levels confer apoptotic resistance to a number of cancer cell lines, ${ }^{20}$ and synthetic
IAP-antagonist compounds show promise as anti-cancer drugs. ${ }^{21}$

Previous genetic screens had identified another IAP family gene, dBruce, as a major anti-apoptotic gene in Drosophila. dBruce encodes a giant (4876 a.a. protein) ubiquitin conjugating enzyme with a BIR domain, and its gain- or loss-offunction conditions modify Grim- and Reaper-induced cell death to a degree similar to dIAP1 alleles. ${ }^{22,23}$ Intriguingly, dBruce alleles do not affect cell death caused by another IAPantagonist, Hid. ${ }^{24}$ Unlike dIAP1 mutants, most somatic cells can survive without $d B r u c e$. However, developing spermatids of $d B r u c e-1-$ flies prematurely activate caspases and undergo apoptosis. ${ }^{23,24}$ In spite of the strong genetic evidence linking dBruce in apoptosis regulation, ubiquitination targets of dBruce have not been identified. The presence of a ubiquitin conjugating (UBC) domain suggests that dBruce ubiquitinates pro-apoptotic proteins, such as Reaper and Grim. However, the difficulty of establishing biochemical links between dBruce and IAP-antagonists has led Vernooy et al. ${ }^{22}$ to suggest that dBruce regulates these proteins indirectly. Challenging that view, here we show the evidence that dBruce directly ubiquitinates Reaper at unconventional acceptor sites. Specifically, dBruce physically interacts with Reaper and the absence of $d B r u c e$ leads to higher Reaper protein levels. Overexpression of the full-length dBruce strongly suppressed a Reaper-induced cell death phenotype, whereas this phenotype was only weakly suppressed by a truncated dBruce line lacking the UBC domain. Unexpectedly, the level

\footnotetext{
${ }^{1}$ Department of Cell Biology, New York University School of Medicine, New York, NY, USA

${ }^{*}$ Corresponding author: HD Ryoo, Department of Cell Biology, NYU School of Medicine, 550 First Avenue, New York, NY 10016, USA. Tel: +212 263 7257;

Fax: + 212263 8139; E-mail: hyungdon.ryoo@nyumc.org

Keywords: apoptosis; ubiquitin; IAP; Reaper; dBruce

Abbreviations: dIAP1, Drosophila Inhibitor of Apoptosis 1; IBM, IAP Binding Motif; GH3, Grim Helix 3; BIR, Baculovirus IAP Repeat; UBC, Ubiquitin Conjugating (domain); Dark, Drosophila Apaf1-rēlated killer

Received 08.2.11; revised 29.7. 11 ; accépted 29.7.11; Edited by E Baehrecke; published online 02.9.11
} 

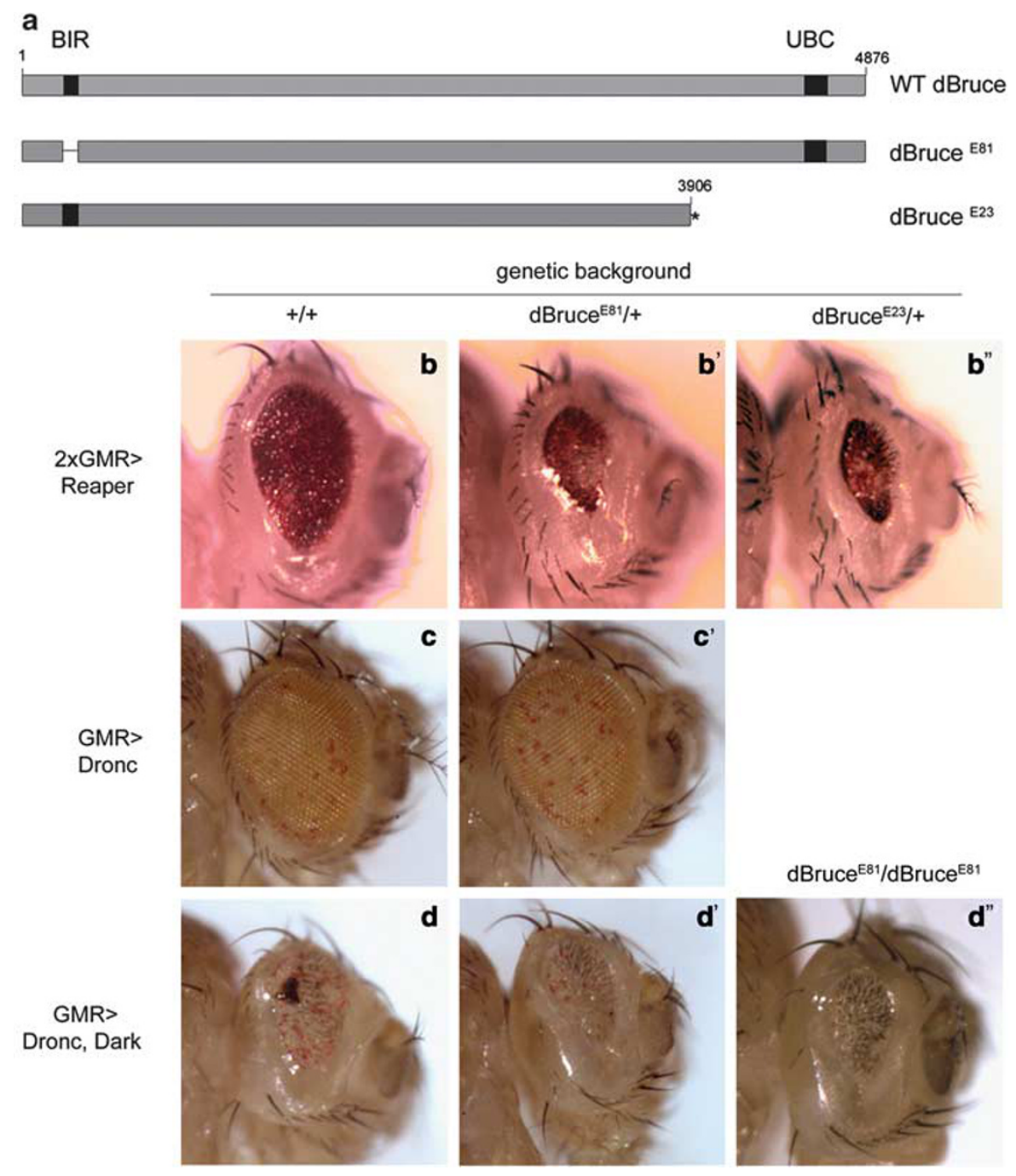

Figure 1 dBruce loss-of-function enhances Reaper-induced cell death in the eye, but not that caused by Reaper-independent apoptosome activation. (a) A schematic diagram of the dBruce primary structure, containing a BIR domain near the $\mathrm{N}$-terminus, and a UBC domain near the $\mathrm{C}$-terminus. $d B$ ruce ${ }^{E 81}$ allele contains an in-frame deletion that eliminates the BIR domain, ${ }^{25}$ whereas the $d B r u c e^{E 23}$ allele contains a premature stop codon that precedes the UBC domain. (b) Partial eye ablation caused by reaper overexpression during eye development $(\mathbf{b})$, is enhanced in both, the $d B r u c e^{E 81} /+$ background $\left(\mathbf{b}^{\prime}\right)$, and the $d B r u c e^{E 23} /+$ background $\left(\mathbf{b}^{\prime \prime}\right)$. On the other hand, $d B r u c e^{E 81} /+$ condition does not impact the phenotype caused by expression of the apical caspase Dronc in the eye field (c and $\mathbf{c}^{\prime}$ ). Here, the uneven distribution of eye pigments is due to eye pigment cell death. The combined expression of the apoptosome components, Dronc and Dark causes a partial eye ablation (d), which is not affected in a $d B r u c e^{E 81 /}+$ background $\left(\mathbf{d}^{\prime}\right)$. Additionally, no enhancement of the small eye phenotype owing to Dark and Dronc co-expression is observed in $d B r u c e E 81 / d B r u c e E 81$ homozygous

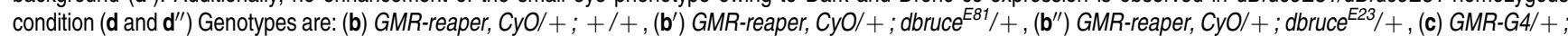

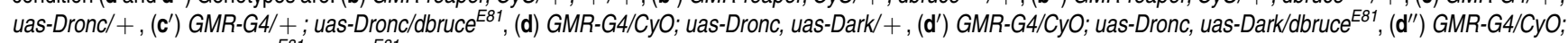
uas-Dronc, uas-Dark, dbruce ${ }^{E 81} /$ dbruce $^{E 81}$

of a mutant form of Reaper that lacks all internal lysines, thereby deficient of conventional ubiquitin acceptor sites, is also elevated in $d B r u c e$ mutants. Furthermore, this form of Reaper is ubiquitinated and the extent of ubiquitination is reduced in $d B r u c e$ knocked down cells, indicating that dBruce tags Reaper with ubiquitins on non-lysine acceptor sites.

\section{Results}

Reaper-induced cell death is modified by loss or gain of dBruce function. dBruce encodes a large protein with a $\mathrm{BIR}$ domain near the N-terminus, and a UBC domain near the C-terminus (Figure 1a). To investigate the function of this gene, we primarily used two loss-of-function alleles: the E81 allele has an in frame deletion that takes out the BIR domain, ${ }^{23}$ and the E23 allele has a stop codon that would not translate the UBC domain (Figure 1a and Materials and Methods). As previously reported with other loss-of-function alleles, ${ }^{24}$ these two alleles behaved similarly in dominantly enhancing the reaper overexpression phenotype in the fly eye (Figures $\left.1 b-b^{\prime \prime}\right)$. To test the specificity of dBruce function, we performed genetic interaction assays between the $d B r u c e$ alleles and downstream caspases of Drosophila. Unlike the reaper-induced phenotype, dBruce $e^{E 81} /+$ condition did not affect the pigment cell death phenotype 

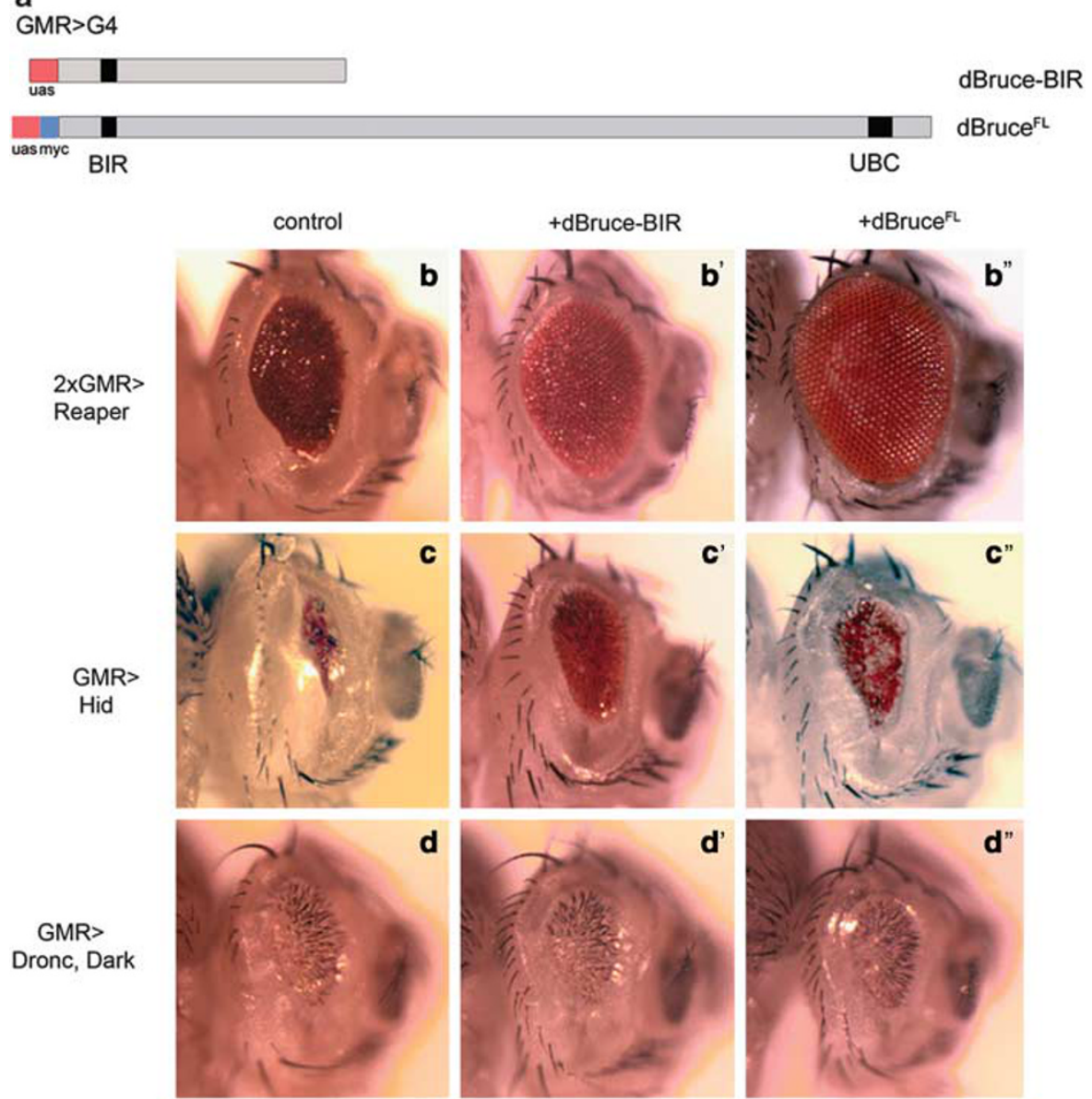

Figure 2 Overexpression studies reveal the importance of both the N-terminal and C-terminal sequences of $d B r u c e$. (a) A scheme showing the $d B$ ruce contructs used to rescue cell-death induced by Reaper, Hid, or Dronc and Dark together. The $d B r u c e-B I R$ construct ${ }^{25}$ contains the first 1622 a.a. residues of dBruce, which includes the BIR but not the UBC motif. The full-length $d B r u c e$ contains the complete sequence. (b) Reaper expression in the eye causes partial eye ablation, and this is partially rescued by the $d B r u c e-B I R$ construct $\left(\mathbf{b}^{\prime}\right)$. Notably, full suppression is obtained only when the full-length $d B r u c e$ is co-expressed $\left(\mathbf{b}^{\prime \prime}\right)$. The small eye phenotype induced by Hid overexpression (c) is also mildly suppressed by the $d B r u c e-B I R$ construct $\left(\mathbf{c}^{\prime}\right)$. In contrast to Reaper, however, the full-length $d B r u c e$ did not fully rescue the Hid-induced eye phenotype $\left(\mathbf{c}^{\prime \prime}\right)$. Co-expression of the apoptosome components Dronc and Dark also led to a partial eye ablation (d), which was neither modified by the co-expression of $d B r u c e-B I R\left(\mathbf{d}^{\prime}\right)$ nor the full-length dBruce (d'). Genotypes: (b) GMR-G4, ey-flp/ +; GMR-Rpr,CyO/Sco; + I+, (b') GMR-G4, ey-flp/ +; GMR-Rpr,CyO/uas-dBruce-BIR;

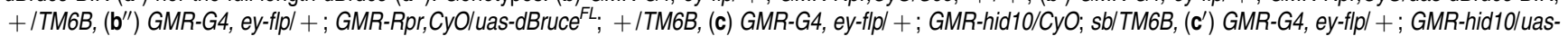
dBruce-BIR; sb/TM6B, (c') GMR-G4, ey-flp/ +; GMR-hid10/uas-dBruce FL; sb/TM6B, (d) GMR-G4/CyO; uas-Dronc, uas-Dark/TM6B, (d') GMR-G4/uas-dBruce-BIR; uasDronc, uas-Dark/TM6B and (d') GMR-G4/uas-dBruce ${ }^{F L}$; uas-Dronc, uas-Dark/TM6B

caused by the overexpression of the initiator caspase Dronc (Figures $\left.1 c-c^{\prime}\right)$. The results were further validated in a genetic condition where caspases were activated by the co-expression of Dronc and its adaptor, Drosophila apaf1related killer (Dark). ${ }^{25}$ The combined expression of Dronc and Dark in the developing eye imaginal discs generates a potent eye ablation phenotype owing to the assembly of the apoptosome, an oligomeric complex of Dark that activates Dronc for apoptosis, ${ }^{26}$ providing a way to activate caspases independent of the IAP-antagonists. This phenotype was neither affected by $d B r u c e^{E 81} /+$ nor by $d B r u c e^{E 81}-/-$ conditions (Figures $1 d-d^{\prime \prime}$ ). These findings suggest that dBruce regulates apoptotic events upstream of the apoptosome.

To examine the effects of dBruce overexpression, we employed two dBruce transgenes, one that can express a truncated form of $d B r u c e$ that retains the BIR domain
(dBruce-BIR), ${ }^{26}$ and the other encoding the full-length $d B r u c e$ (dBruce $^{F L}$ ) (Figure $2 \mathrm{a}$ and Materials and Methods). Co-expression of $d B r u c e-B I R$ only moderately suppressed the reaper overexpression phenotype, whereas the $d B r u c e^{F L}$ completely rescued that phenotype under otherwise similar conditions (Figures $\left.2 \mathrm{~b}-\mathrm{b}^{\prime \prime}\right)$. dBruce-BIR also weakly suppressed the eye ablation phenotype caused by hid overexpression (Figures $2 c$ and $c^{\prime}$ ). Unlike the reaper-inflicted eye phenotype, however, $d B r u c e^{F L}$ did not suppress the hid phenotype any better than that by $d B r u c e-B I R$ (Figure $\left.2 b^{\prime \prime}\right)$. In addition, no suppression was observed when either $d B r u c e-B I R$ or $d B r u c e^{F L}$ was co-expressed with the apoptosome components, Dronc and Apaf1 (Figures $\left.2 d-d^{\prime \prime}\right)$. This is in agreement with the idea that dBruce does not regulate caspases directly, and point to IAP-antagonists as likely targets of $d B r u c e$. Although the anti-apoptotic function can be partly attributed to the dBruce BIR domain, the sequences 
a

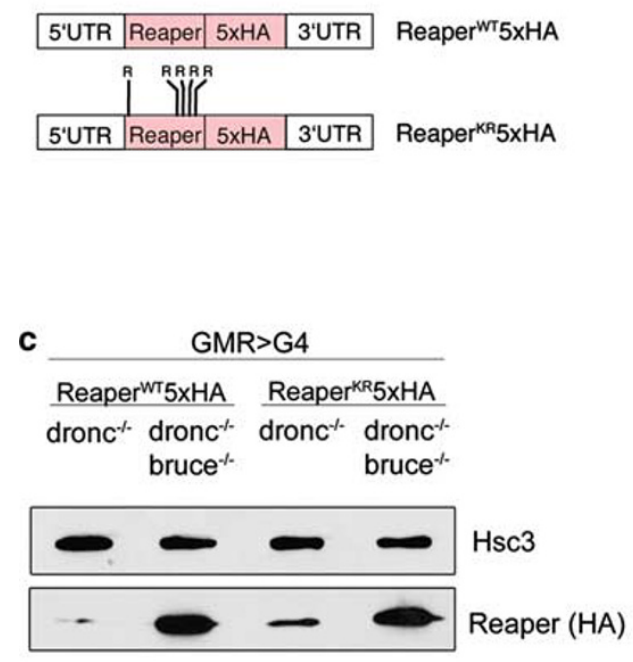

b
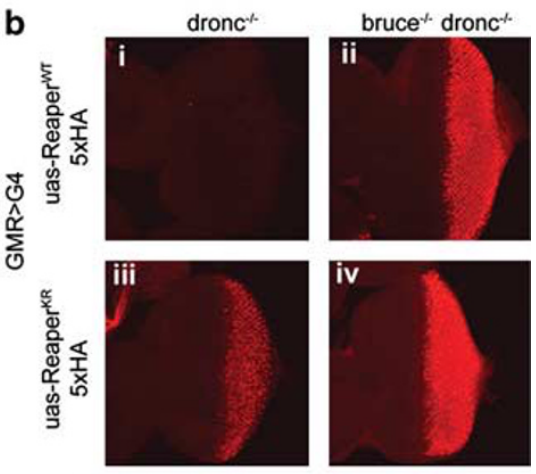

d dronc $^{-1}$ dronc ${ }^{-1}$

bruce $^{- \text {- }}$
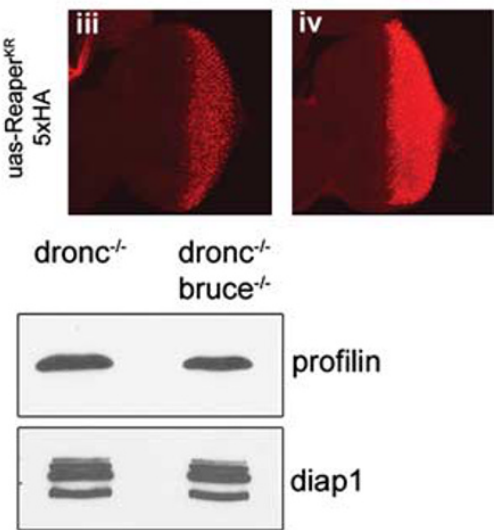

Figure 3 dBruce loss-of-function condition enhances Reaper protein levels, even in the absence of lysine residues. (a) A graphic scheme of the Reaper transgenes used in this study. The Reaper coding sequence was cloned in frame with a $5 \times \mathrm{HA}$ tag to generate a fusion protein, flanked by the $5^{\prime}$ and $3^{\prime} \mathrm{UTR}$ regions of the Reaper transcription unit. The transgenes were targeted for insertion in a predetermined genomic locus (51C). Reaper-KR has all internal lysine residues (at positions 20, 52, 56, 59 and 62) mutated to arginine. (b) Reaper protein levels as assessed by anti-HA immunolabeling (red). All experiments were performed in a dronc ${ }^{129}-1-$ background to allow the survival of Reaper expressing cells. The levels of wild-type Reaper (Reaper-WT) tagged with $5 \times$ HA are visualized in (i) and (ii). Reaper-KR levels similarly visualized with anti-HA are shown in (iii) and (iv). In the $d B r u c e^{E 81}-/$ - background, higher levels of both Reaper-WT and Reaper-KR are detected ((ii) and (iv)) as compared with the dBruce + background ((i) and (iii)). Genotypes are: (i) GMR-G4/uas-reaper ${ }^{W T} 5 \times H A$; dronc ${ }^{129} /$ dronc $^{129}$ (ii) GMR-G4/uas-reaper ${ }^{W T} 5 \times H A$; dronc ${ }^{129}$, dbruce ${ }^{E 81} /$ dronc $^{129}$, dbruce $^{E 81}$ (iii) GMR-G4/uas-reaper ${ }^{K R} 5 \times H A$; drond ${ }^{129} / d r o n c^{129}$ and (iv) GMR-G4/uas-reaper ${ }^{K R} 5 \times H A$; drond ${ }^{129}$, dbruce ${ }^{E 81} /$ dronc $^{129}$, dbruce ${ }^{E 81}$. (c) Confirmation of results in (b) through western blot. Extracts were prepared from $\sim 30$ eye imaginal discs of the specific genotypes as in (b). Reaper levels as assessed through anti-HA blot (lower panel) can be compared with a control of anti-Hsc3 blot (upper panel). (d) Endogenous dIAP1 levels are not altered in the dBruce -I- background. Anti-dlAP1 western blot (lower panel) can be compared with anti-Profilin controls (upper panel). Genotypes: dronc ${ }^{129} / \mathrm{dronc}^{129}$ (left lane) and $\mathrm{dronc}^{129}, \mathrm{dbruce}^{E 81} / \mathrm{dronc}^{129}$, dbruce ${ }^{E 81}$ (right lane)

outside of the BIR domain (possibly the UBC domain) are also required for $d B r u c e$ 's full anti-apoptotic function.

dBruce regulates the levels of wild-type and lysinedeficient mutant Reaper. Although Vernooy et al. $^{22}$ had previously speculated that Reaper is not a direct target of dBruce, the specificity of the genetic interactions prompted us to revisit this issue. As a first step, we analyzed the effect of dBruce mutations on the levels of Reaper protein expressed in the developing eye imaginal discs. To visualize ectopically expressed Reaper proteins, we generated a transgenic UAS-Reaper line with five tandem repeats of the $\mathrm{HA}$ epitope $(5 \times \mathrm{HA})$ added to its $\mathrm{C}$-terminus (Figure $3 a$ ). In addition, we generated a similar UAS-Reaper line, in which all lysine residues were replaced with arginines (henceforth referred to as Reaper-KR), thereby eliminating conventional ubiquitin acceptor sites within this protein (Figure 3a). The HA epitope does not introduce any lysines. To ensure equivalent levels of transcription, both transgenes were targeted to the same genomic locus through the phiC31 integrase system (see Materials and Methods). Reaper protein expression analysis was performed in the dronc mutant background, ${ }^{27}$ which prevents caspase activation and cell death that otherwise complicates the assessment of Reaper protein levels (Supplementary Figure S1). Immunostaining against HA under these conditions showed faint signals from the wildtype Reaper protein (Reaper-WT), whereas a significantly higher signal was detected for Reaper-KR (Figure 3b, compare (i) and (iii). These results indicate that Reaper protein levels are affected by internal lysine residues, most likely due to ubiquitin-mediated protein degradation.

To test whether $d B r u c e$ affects Reaper levels, we examined the levels of HA-tagged Reaper expression in a dBruce mutant background. Consistent with the idea that dBruce promotes Reaper degradation, we found a significant increase in Reaper-WT levels in the $d B r u c e^{E 81}-/$ - condition (Figure 3b, compare (i) and (ii)). Unexpectedly, we found that Reaper-KR levels also increase under this condition, although to a lesser degree than Reaper-WT (Figure 3b, compare (iii) and (iv)). To rule out the possibility of an immunostaining artifact or epitope masking, we confirmed these results by western blot analysis of Reaper-HA levels from eye imaginal disc extracts (Figure $3 c$ ). In validation of our immunostaining results, we found that extracts from $d B$ Buce $-/$ - tissues have more than 10 times as much wild-type Reaper $(P=0.0417)$, and $\sim 3$ times as much Reaper-KR levels $(P=0.0497)$, as $d B r u c e+$ tissues (Figures $3 c$ and $4 \mathrm{e}$ ).

Reaper and dBruce physically interact through Reaper's N-terminal and GH3 motifs. The observation that Reaper-KR levels increase in dBruce -/- tissues raised 
a

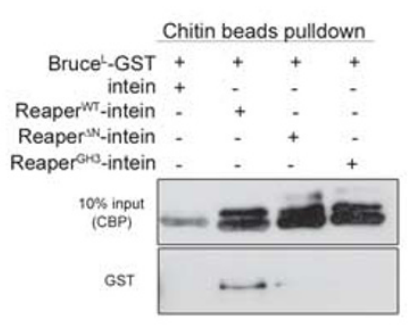

C

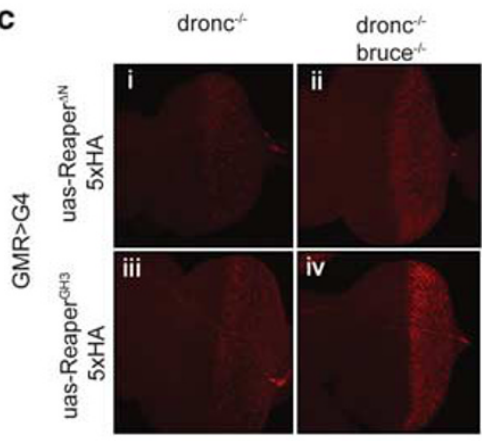

b

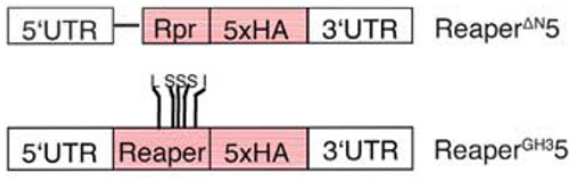

d

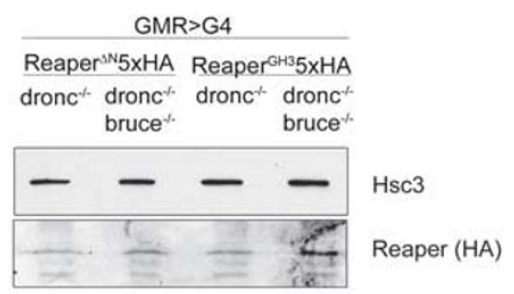

e

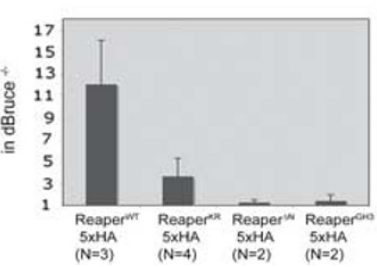

Figure 4 dBruce and Reaper physically interact. (a) A portion of dBruce containing the BIR domain fused with GST (Bruce -GST, residues 1-481) purified and eluted from glutathione beads was incubated with purified Reaper-CBD bound to chitin beads (or CBD alone). Physical interaction was detected between dBruce and Reaper ${ }^{\text {WT }}$-CBD. Mutations in Reaper's N-terminus (Reaper ${ }^{\Delta \mathrm{N}}$ ) or Reaper's GH3 domain (Reaper ${ }^{\mathrm{GH} 3}$ ) abolish this interaction in otherwise identical conditions. (b) A schematic diagram of the Reaper mutant transgenes targeted to the $51 \mathrm{C}$ locus. Reaper ${ }^{\Delta \mathrm{N}}$ has a deletion encompassing the IBM motif (residues 2-8), while Reaper ${ }^{\mathrm{GH} 3}$ has mutations in the GH3 motif as follows: ${ }^{32} \mathrm{~W}$ to $\mathrm{L},{ }^{34} \mathrm{~F}$ to $\mathrm{S},{ }^{35} \mathrm{~L}$ to $\mathrm{S},{ }^{36} \mathrm{~A}$ to $\mathrm{S}$ and ${ }^{36} \mathrm{~V}$ to I. (c) The in vivo levels of Reaper ${ }^{\Delta \mathrm{N}}$ ((i) and (ii)) and Reaper ${ }^{\text {GH3 }}$ ((iii) and (iv)) proteins ectopically expressed in eye imaginal discs as assessed through anti-HA immunolabeling (red). The levels can be compared between dbruce + ((i) and (iii)) and dbruce -I- ((ii) and (iv)) conditions. Genotypes: (i) GMR-G4/uas-reaper ${ }^{1 N} 5 \times H A$; dronc $c^{129} / d_{\text {dronc }}{ }^{129}$, (ii) GMR-G4/uas-reape ${ }^{1 N} 5 \times H A$; dronc $c^{129}$, dbruce ${ }^{E 81} /$ dronc $^{129}$, dbruce E81 (iii) GMR-G4/uasreaper $^{G H 3} 5 \times H A$; drond $c^{29} /$ dronc $^{129}$ and (iv) GMR-G4/uas-reaper ${ }^{G H 3} 5 \times H A$; dronc ${ }^{129}$, dbruce ${ }^{E 81} /$ drond $^{129}$, dbruce ${ }^{E 81}$. (d) Confirmation of results in (c) through western blot analysis. Extracts from around 30 eye imaginal discs for each genotype were probed through anti-HA antibody (lower panel) and compared with anti-Hsc3 controls (upper panel). (e) Quantification of western blot results shown in (c). y axis shows estimated Reaper protein levels in dronc $-/-, d b r u c e^{E 81}-/-$ imaginal disc extracts divided by those in control dronc $-/$ - extracts. $\mathrm{N}$ indicates the number of independent experiments performed, while the error bar shows standard deviation

two possibilities: in an 'indirect model' as proposed by Vernooy et al., ${ }^{22}$ dBruce would affect an unknown intermediate protein whose function, in turn, affects Reaper levels. Among others, we considered dIAP1 as a potential mediator of dBruce's 'indirect effects' on Reaper, as previous studies have shown that dIAP1 acts as a ubiquitin ligase that can promote the degradation of Reaper. ${ }^{13}$ However, dIAP1 levels did not change in $d B r u c e$ loss-of-function conditions (Figure 3d).

By contrast, a 'direct model' can be considered in which dBruce promotes ubiquitination of Reaper directly, but utilizes non-lysine residues as ubiquitin acceptor sites. In the latter case, mutating all internal lysine residues in the Reaper sequence would not be sufficient to prevent dBruce ubiquitination of Reaper. In a first step to examine the 'direct model', we tested possible physical interactions between dBruce and Reaper in an in vitro pull down assay using chitin binding domains (see Materials and Methods). Specifically, we found that recombinant Bruce-GST (residues 1-481 that include the BIR domain) associated with Reaper-WT in this assay (Figure 4a). We also performed binding assays with Reaper mutants that either lack the $\mathrm{N}$-terminal IAP binding motif (IBM), or have mutations in the central helical domain (GH3). IBM is a motif that can bind to a related BIR domain present in $\mathrm{dlAP}^{28}$ and the $\mathrm{GH} 3$ has self-associating properties that promote the formation of multimeric IAP-antagonist complexes. ${ }^{29}$ Neither of these mutant proteins interacted with dBruce in vitro (Figure 4a). This result indicates that the BIR domain of dBruce has direct affinity for Reaper in vitro, and that this interaction is specific. These in vitro results are also consistent with the $d B r u c e-B I R$ overexpression results in vivo (Figure 2), where reaper-and hid-induced cell death was moderately suppressed.

To test if dBruce regulates Reaper through the IBM and GH3 motifs in vivo, we generated transgenic constructs of these Reaper mutants with $5 \times \mathrm{HA}$ tags at their $\mathrm{C}$-terminus (Figure $4 \mathrm{~b}$ ), and targeted them for insertion into a predetermined genomic locus (51C). Consistent with the idea that dBruce does not bind to these Reaper mutants in vivo, we observed no significant alteration of these Reaper protein levels in the presence or absence of dBruce (Figures $4 \mathrm{c}$ and $d$ ). We have quantified these results after western blot, and found that the stabilization fold is 1.25 for Reaper $\Delta \mathrm{N}$ and 1.4 for Reaper-GH3, and the differences observed are not statistically significant $(P=0.4096$ and $P=0.5024$, respectively; see Figure 4e).

Reaper-KR is ubiquitinated in vitro, and the abundance of the ubiquitinated species is dependent on dBruce. Our finding that dBruce regulates Reaper levels prompted us to biochemically examine the extent of Reaper ubiquitination in cultured Drosophila S2R + cell extracts. To 


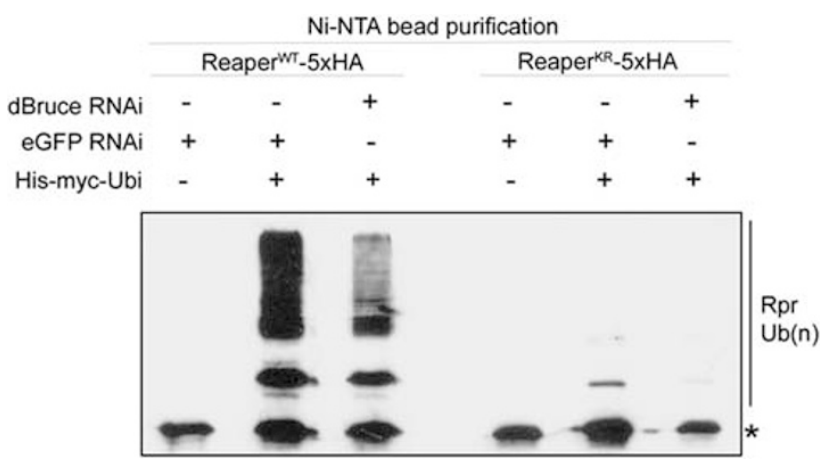

Figure 5 dBruce dependent ubiquitination of wild-type and lysine-deficient Reaper proteins. In order to purify ubiquitinated Reaper proteins from cultured cells, $\mathrm{S} 2 \mathrm{R}+$ cells were transfected with Reaper- $5 \times \mathrm{HA}$ and His-tagged ubiquitin expression plasmids. Cells were treated with a proteasome inhibitor before his-ubiquitin-tagged proteins were purified from these extracts using Ni-NTA resin. The Reaper protein was detected through western blot with anti-HA antibody. The asterisk indicates the band corresponding to unmodified Reaper protein that was retained through unspecific matrix interaction. Higher molecular weight bands of Reaper-WT-5 $\times$ HA were detected only when His-ubiquitin was co-transfected (lanes 2, 3, 5 and 6), indicative of ubiquitin conjugation. His-ubiquitin dependent higher molecular weight Reaper-KR was also detected, although at a lower extent (lane 5). Knockdown of $d B r u c e$ by RNAi decreases the abundance of the ubiquitinated species for both Reaper-WT and in Reaper-KR, supporting the idea that dBruce ubiquitinates Reaper on non-lysine residues

keep cells alive after Reaper transfection, we generated an inducible $\mathrm{S} 2 \mathrm{R}+$ cell line that expresses the baculovirus caspase inhibitor p35. ${ }^{30}$ We transfected into these cells $5 \times \mathrm{HA}$ tagged Reaper with or without His-tagged ubiquitin. Purification of the ubiquitin-tagged proteins with Ni-NTA beads from these extracts, and subsequent detection of Reaper with anti-HA antibody revealed a ladder of higher molecular weight bands, corresponding to ubiquitinated Reaper species (Figure 5). These higher molecular weight species appeared only when His-ubiquitin was cotransfected (for example, compare between lanes 1 and 2 in Figure 5), indicating that they emerge as a result of ubiquitin conjugation. Consistent with the idea that Reaper is extensively ubiquitinated, wild-type Reaper exhibited a very prominent ubiquitination profile (Figure 5, compare lanes 1 and 2). Interestingly, Reaper-KR showed a more modest but unequivocal ubiquitination pattern (Figure 5, compare lanes 4 and 5). These results confirmed our hypothesis that Reaper must contain non-lysine ubiquitin acceptor sites.

There are a number of known ubiquitin-acceptor sites other than lysines. These include cysteines, serines, threonines and the N-terminal amino group. ${ }^{31-34}$ To examine the roles of these unconventional acceptor sites, we made Reaper-HA transgenes that have combinations of these residues mutated, on top of the five lysine mutations. These mutants made proteins that still appeared to have residual ubiquitin conjugation (Supplementary Figure S2). This result suggests that either another combination of residues might be responsible for accepting the ubiquitin moiety, or ubiquitination occurs at the N-terminal amino group. Testing the latter idea is impossible, as manipulating this $\mathrm{N}$-terminus also abolishes Reaper's ability to bind BIR domains of its ubiquitinating enzymes. ${ }^{28}$
To determine whether dBruce is responsible for the emergence of non-lysine conjugated ubiquitin chains on Reaper, we performed the Reaper ubiquitination analysis after knockdown of dBruce function. We found that dBruce RNAi treatment results in a decrease in the abundance of the ubiquitinated species, both with Reaper-WT and Reaper-KR (Figure 5, compare lanes 2 and 3, 5 and 6, respectively). The fact that these bands do not completely disappear may be owing to an incomplete knockdown of dBruce, or alternatively, owing to other ubiquitination enzymes that target Reaper. Taken together, these results support the idea that dBruce directly regulates Reaper protein levels by ubiquitinating it at lysine and non-lysine residues.

\section{Discussion}

As it was first identified, dBruce has attracted significant scientific interest as one of the strongest modifiers of apoptosis in Drosophila. Surprisingly, little is known about the molecular function and substrates of dBruce. Here, we established Reaper as a direct target of dBruce in flies. Our structure/function analysis of Reaper revealed the requirement of both the N-terminal IAP-binding domain and the central helical domain (GH3) for directly binding to dBruce. The IAP-Binding Motif (IBM) binds to the BIR domain of dIAP $1,{ }^{28}$ and its requirement suggests that Reaper binds to $a$ similar BIR motif present in dBruce. The GH3 motif has been recently characterized as a multimer-forming interface between IAP-antagonists, ${ }^{29}$ which leads us to suspect that dBruce specifically recognizes active Reaper multimers, but not monomers. A similar GH3 motif is present in Grim, which genetically interacts with dBruce. ${ }^{24}$ On the other hand, $d B r u c e$ does not show strong genetic interactions with Hid, which lacks a GH3 motif. In mammals, the dBruce homolog BRUCE (otherwise known as APOLLON) can bind and ubiquitinate a mammalian IAP-antagonist, Smac. ${ }^{35,36}$ Noteworthy is the fact that Smac also forms dimers in vitro. ${ }^{37}$ There are a number of other IAP-antagonists in mammals, and it remains to be seen whether their susceptibility to BRUCE correlates with their ability to form multimeric complexes.

Intriguingly, our data indicate that dBruce ubiquinates Reaper on non-lysine ubiquitin acceptor sites. This is in contrast to dIAP1 and X-linked IAP, which are thought to ubiquitinate Reaper and caspases strictly on lysine side chains. ${ }^{13,15}$ Our results nicely explain a previously published observation that dBruce overexpression can somehow suppress apoptosis initiated by lysine-deficient Reaper. ${ }^{22}$ But unlike the conclusions drawn by those authors, we conclude that the inhibition is direct.

In our study, we did not find any evidence that $d B r u c e$ regulates caspases directly. Specifically, $d B r u c e$ does not affect the cell killing activity of the initiator caspase Dronc. The result is consistent with the observation that $d B r u c e$ does not strongly affect Hid function, which also triggers apoptosis through the activation of initiator and effector caspases. ${ }^{22}$ These results have implications on the potential mechanisms of premature caspase activation that occurs in dBruce -/spermatids. A simple model would suggest that, as in somatic tissues, dBruce primarily targets Grim and Reaper in differentiating spermatids, and the deregulation of these 
IAP-antagonists lead to premature caspase activation. Alternatively, developing spermatids may have additional dBruce co-factors that allow dBruce to target caspases. In somatic tissues, it appears that $d B r u c e$ regulates apoptosis primarily by acting as an IAP-antagonist inhibitor. As synthetic IAPantagonists are actively being developed as anti-cancer compounds, the endogenous BRUCE levels may contribute to a particular cell's sensitivity or resistance to these compounds.

\section{Materials and Methods Generation of the p35 inducible cell line (S2R + p35). Xhol/Notl sites were used to subclone the full-length $\mathrm{p} 35 \mathrm{CDNA}^{30}$ into the pMK33-HS vector, which contains a methallothionein promotor. This plasmid was transfected into S2R + cells, and hygromycin resistance was used to isolate a stable insertion line.}

Cell culture and cell transfection. Cells were transfected using effectene (Qiagen, Valencia, CA, USA). To knock down dBruce, $15.6 \times 10^{6} \mathrm{~S} 2 \mathrm{R}+\mathrm{p} 35$ cells in $75 \mathrm{~cm}^{2}$ T-flasks were transfected with $19.5 \mu \mathrm{g}$ dsRNA, $3.9 \mu \mathrm{g}$ of actin-Gal 4 and $3.9 \mu \mathrm{g}$ uas-dicer2, 7 days before transfection of Reaper plasmids. To induce Reaper expression, cells were re-plated in six-well plates, and transfected with $1 \mu \mathrm{g}$ actinGal4 and $1 \mu \mathrm{g}$ of uas-Reaper plasmids. In order to visualize ubiquitinated proteins, $1 \mu \mathrm{g}$ His-Myc-Ubiquitin plasmid or empty vector was co-transfected. CuSO4 was added to a final concentration of $0.5 \mathrm{mM}$ to induce p35 expression to prevent Reaper from killing transfected cells. 3 days after transfection, cells were treated with $50 \mu \mathrm{M}$ MG132 for $6 \mathrm{~h}$, then collected and resuspended in $150 \mu \mathrm{l}$ lysis buffer (1\% SDS, $10 \mathrm{mM}$ EDTA pH 8.0, $50 \mathrm{mM}$ Tris- $\mathrm{HCl}$ pH 8.0 and $1 \mathrm{mM}$ PMSF) supplemented with $5 \mathrm{mM} \mathrm{NEM}$ and protease inhibitors. Crude extract was sonicated and diluted $1: 10$ in IP dilution buffer $(0.01 \%$ SDS, $1.1 \%$ Triton X-100, $1.2 \mathrm{mM}$ EDTA, $16.7 \mathrm{mM}$ Tris- $\mathrm{HCl} \mathrm{pH} 8.0,150 \mathrm{mM} \mathrm{NaCl}$ and $1 \mathrm{mM}$ PMSF) supplemented with $5 \mathrm{mM}$ NEM and protease inhibitors (Roche, Indianapolis, IN, USA). To purify proteins conjugated with His-tagged ubiquitin, each extract was incubated with $20 \mu \mathrm{l}$ Ni-NTA His-Bind Superflow beads (Novagen, San Diego, CA, USA) for $2 \mathrm{~h}$ at $4{ }^{\circ} \mathrm{C}$ and washed three times in PBS $+20 \mathrm{mM}$ imidazole. SDS loading buffer (containing DTT that releases $\mathrm{Ni}^{2+}$ bound proteins from beads) was added to each sample before boiling for $5 \mathrm{~min}$ at $95^{\circ} \mathrm{C}$ and loading onto a $15 \%$ SDS page gel. The nitrocellulose membranes were autoclaved for 30 minutes after transfer. To visualize Reaper through western blots, rat monoclonal anti-HA (Roche, clone 3F10) was used at 1:500 dilution.

Cloning. Generation of transgenes for fly transformation: to make a full-length dBruce transgene, a 19.7-kb genomic DNA encompassing the locus was cloned from the $\mathrm{CH} 321-36 \mathrm{~F} 16 \mathrm{BAC}$ clone (Pacman Resources) using the recombineering technology. ${ }^{38}$ The construct was designed to have the coding sequence fused in frame with a myc-epitope into a pre-engineered UAS-myc-pACMAN vector. Reaper coding sequences with in frame $5 \times \mathrm{HA}$ tags were cloned into BamHI sites engineered in between Reaper's $5^{\prime}$ UTR and $3^{\prime}$ UTR sequences. Using EcoR1 sites, the engineered cDNAs were moved to the pUAST attB vector and inserted into the $51 \mathrm{C}$ locus using a PhiC31 integrase strain. ${ }^{39}$ Generation of Reaper-CBD fusions and BruceL-GST: to generate recombinant protein fused to the chitin binding domain (CBD), coding sequences of wild-type or mutant Reaper were subcloned into the Nde1 site of the pTYB2 plasmid (N.E.B.), which encodes Reaper fused to the N-terminal to intein-CBD. Using the same approach, mutant Reaper lacking the $\mathrm{N}$-terminal eight amino acids $(\Delta \mathrm{N})$, or with mutations in the $\mathrm{GH} 3$ domain ${ }^{29}$ were subcloned into PTYB2. Bruce ${ }^{\mathrm{L}}$-GST was made by subcloning a PCR product that encodes from the original start codon to amino acid 481, into pGEX 4t-3. These recombinant proteins were expressed in BL21 cells according to standard protocols. His-Myc-Ubiquitin: a plasmid encoding N-terminal $6 \times$ His-Myc fused to mammalian ubiquitin ${ }^{32}$ was obtained from Michele Pagano, and EcoRI/Kpnl sites were used to move the $6 \times$ His-Myc-ubiquitin into the pUAST vector.

Eye imaginal disc western blot quantification. About 30 pairs of eye imaginal discs were dissected for each genotype, and extracts were made in eye disc lysis buffer (10 mM Tris-HCl pH 8.0, $1 \mathrm{mM}$ EDTA pH 8.0, $150 \mathrm{mM} \mathrm{NaCl}$ and $1 \%$ SDS). Crude extracts were centrifuged 10 minutes at maximum speed at $4{ }^{\circ} \mathrm{C}$, and the supernatant was quantified using Bradford assay. Normalized quantities were loaded onto $15 \%$ SDS page gels. Anti-HA antibody (clone 3F10, Roche) was used to assess Reaper levels, and anti-Hsc3 (generated in the lab) or anti-Profilin (Chi1J, Developmental Studies Hybridoma Bank, University of lowa) were used as loading controls.

Fly strains. Genes were overexpressed in Drosophila imaginal discs using the standard Gal4/UAS system. ${ }^{40}$ The Reaper transgenes, as well as the full-length UAS-dBruce flies were generated in this study. uas-Dronc, uas-Dark, ${ }^{26}$ uas-dBruce$B I R,{ }^{24}$ dronc $^{129}$ (Xu et al. ${ }^{27}$ ) and $d B r u c e^{E 81}$ and $d B r u c e^{E 23}$ (Arama et al. ${ }^{23}$ ) were as reported previously. As the molecular nature of the $d B r u c e^{E 23}$ allele had not been reported previously, we sequenced this allele and found an $11 \mathrm{bp}$ (GCCAAGGACAA) deletion in the coding sequence, generating a frameshift at a.a. 3901 , leading to a premature stop codon at a.a. 3906.

Pulldown experiments. $1 \mathrm{ml}$ of induced bacterial extracts of Reaper-CBD (or control plasmids) were bound to $40 \mu$ lo chitin beads (New England Biolabs). Beads were washed three times in binding buffer (PBS1 $\times$ containing $0.1 \%$ Igepal CA-630, Sigma, St. Louis, MO, USA). The amount of bound Reaper was estimated by comparing the intensity of Commassie staining with known amounts of BSA proteins. $10 \mu \mathrm{g}$ of each Reaper-CBD proteins was used for binding, and $1 \mu \mathrm{g}(10 \%)$ was saved for input analysis. Bruce ${ }^{\mathrm{L}}$-GST was purified with glutathione sepharose beads (GE Healthcare), and the fusion protein was eluted using $20 \mathrm{mM}$ glutathione pH8.0 solution. Eluted Bruce ${ }^{L}$-GST was quantified as described above, and $1 \mu \mathrm{g}$ was used for each incubation. An aliquot of $10 \%$ input was saved for input analysis.

$10 \mu \mathrm{g}$ of Reaper-CBD or control protein bound to chitin beads was incubated with $1 \mu \mathrm{g}$ of eluted Bruce ${ }^{L}$-GST in a final volume of $300 \mu \mathrm{l}$ in binding buffer (PBS1 $\times$ containing $0.1 \%$ Igepal CA-630, Sigma) for $3 \mathrm{~h}$ at $4{ }^{\circ} \mathrm{C}$. Beads were washed three times in binding buffer. A volume of $30 \mu \mathrm{l}$ of $3 \%$ SDS was added to the beads, and the supernatant was loaded onto $10 \%$ SDS gels. Mouse anti-CBP 1:1000 antibody was used to detect Reaper-CBD fusions (Santa Cruz Biotechnology, Santa Cruz, CA, USA), and affinity-purified rabbit anti-GST antibody (ICL, Newberg, OR, USA) 1:2000 was used to detect Bruce ${ }^{L}$-GST.

\section{Conflict of Interest}

The authors declare no conflict of interest.

Acknowledgements. We thank Eli Arama, Sally Kornbluth, Michele Pagano and Hermann Steller for fly stocks and plasmids, Michele Pagano for advice on protein ubiquitination analysis, Eli Arama, Hermann Steller and Jessica Treisman for insightful comments and suggestions. This work was partly supported by a postdoctoral fellowship from Fundação para a Ciência e Tecnologia, Portugal, to $\mathrm{CD}$, and the NIH grant R01GM079425 and its ARRA Supplement to HDR.

1. Jacobson MD, Weil M, Raff MC. Programmed cell death in animal development. Cell 1997; 88: 347-354

2. Hanahan D, Weinberg R. The hallmarks of cancer. Cell 2000; 100: 57-70.

3. White K, Grether ME, Abrams JM, Young L, Farrell K, Steller H. Genetic control of programmed cell death in Drosophila. Science 1994; 264: 677-683.

4. Ryoo HD, Baehrecke EH. Distinct death mechanisms in Drosophila development. Curr Opin Cell Biol 2010; 22: 889-895.

5. Orme M, Meier P. Inhibitor of apoptosis proteins in Drosophila: gatekeepers of death. Apoptosis 2009; 14: 950-960.

6. Hay BA, Wassarman DA, Rubin GM. Drosophila homologs of baculovirus inhibitor of apoptosis proteins function to block cell death. Cell 1995; 83: 1253-1262.

7. Goyal L, McCall K, Agapite J, Hartwieg E, Steller H. Induction of apoptosis by Drosophila reaper, hid and grim through inhibition of IAP function. EMBO J 2000; 19: 589-597.

8. Ryoo HD, Bergmann A, Gonen H, Ciechanover A, Steller H. Regulation of Drosophila IAP1 degradation and apoptosis by reaper and ubcD1. Nat Cell Biol 2002; 4: 432-438.

9. Yoo SJ, Huh JR, Muro I, Yu H, Wang L, Wang S et al. Hid, Rpr and Grim negatively regulate DIAP1 levels through distinct mechanisms. Nat Cell Biol 2002; 4: 416-424.

10. Ditzel M, Wilson R, Tenev T, Zachariou A, Paul A, Deas E et al. Degradation of DIAP1 by the N-end rule pathway is essential for regulating apoptosis. Nat Cell Biol 2003; 4 : $445-450$.

11. Yokokura T, Dresnek D, Huseinovic N, Lisi S, Abdelwahid E, Bangs $P$ et al. Dissection of DIAP1 functional domains via a mutant replacement strategy. J Biol Chem 2004; 279: 52603-52612.

12. Wilson R, Goyal L, Ditzel M, Zachariou A, Baker DA, Agapite J et al. The DIAP1 RING finger mediates ubiquitination of Dronc and is indispensable for regulating apoptosis. Nat Cell Biol 2002; 4: 445-450. 
13. Olson MR, Holley CL, Yoo SL, Huh JR, Hay BA, Kornbluth S. Reaper is regulated by IAP-mediated ubiquitination. J Biol Chem 2003; 278: 4028-4034.

14. Herman-Bachinsky Y, Ryoo HD, Ciechanover A, Gonen H. Regulation of the Drosophila ubiquitin ligase Diap1 is mediated via several distinct ubiquitin system pathways. Cell Death Differ 2007; 14: 861-871.

15. Ditzel M, Broemer M, Tenev T, Bolduc C, Lee TV, Rigbolt KT et al. Inactivation of effector caspases through nondegradative polyubiquitylation. Mol Cell 2008; 32: 540-553.

16. Broemer M, Tenev T, Rigbolt KT, Hempel S, Blagoev B, Silke J et al. Systematic in vivo RNAi analysis identifies IAPs as NEDD8-E3 ligases. Mol Cell 2010; 40: 810-822.

17. Du C, Fang M, Li Y, Li L, Wang X. Smac a Mitochondrial Protein that Promotes Cytochrome c-Dependent Caspase Activation by Eliminating IAP Proteins. Cell 2000; 102: 33-42.

18. Verhagen A, Ekert PG, Pakusch M, Silke J, Connolly LM, Reid GE et al. Identification of DIABLO, a Mammalian Protein that Promotes Apoptosis by Binding to and Antagonizing IAP Proteins. Cell 2000; 102: 43-54.

19. Garcia-Fernandez M, Kissel H, Brown S, Gorenc T, Schille AJ, Rafii S et al. Sept4/ARTS is required for stem cell apoptosis and tumor suppression. Genes Dev 2010; 24: 2282-2293.

20. Tamm I, Kornblau SM, Segall H, Krajewski S, Welsh K, Kitada S et al. Expression and prognostic significance of IAP-family genes in human cancers and myeloid leukemias. Clin Cancer Res 2000; 6: 1796-1803

21. Dynek JN, Vucic D. Antagonists of IAP proteins as cancer therapeutics. Cancer Lett 2010 e-pub ahead of print 2 Agust 2011; doi:10.1016/j.canlet.2010.06.013.

22. Vernooy SY, Chow V, Su J, Verbrugghe K, Yang J, Cole S et al. Drosophila Bruce can potently suppress Rpr- and Grim-dependent but not Hid-dependent cell death. Curr Biol 2002; 12: 1164-1168.

23. Arama E, Agapite J, Steller H. Caspase activity and a specific cytochrome $\mathrm{C}$ are required for sperm differentiation in Drosophila. Dev Cell 2003; 4: 687-697.

24. Kaplan Y, Gibbs-Bar L, Kalifa Y, Feinstein-Rotkopf Y, Arama E. Gradients of a ubiquitin E3 ligase inhibitor and a caspase inhibitor determine differentiation or death in spermatids. Dev Cell 2010; 19: 160-173.

25. Yu X, Wang L, Acehan D, Wang X, Akey CW. Three-dimensional structure of a double apoptosome formed by the Drosophila Apaf-1 related killer. J Mol Biol 2006; 355: 577-589.

26. Shapiro PJ, Hsu HH, Jung H, Robbins ES, Ryoo HD. Regulation of the Drosophila apoptosome through feedback inhibition. Nat Cell Biol 2008; 10: 1440-1446.
27. Xu D, Li Y, Arcaro M, Lackey M, Bergmann A. The CARD-carrying caspase Dronc is essential for most, but not all, developmental cell death in Drosophila. Development 2005; 132: $2125-2134$

28. Wu JW, Cocina AE, Chai J, Hay BA, Shi Y. Structural analysis of a functional DIAP1 fragment bound to grim and hid peptides. Mol Cell 2001; 8: 95-104.

29. Sandu C, Ryoo HD, Steller H. Drosophila IAP antagonists form multimeric complexes to promote cell death. J Cell Biol 2010; 190: 1039-1052.

30. Clem RJ, Fechneimer M, Miller LK. Prevention of apoptosis by a baculovirus gene during infection of insect cells. Science 1991; 254: 1388-1390.

31. Reinstein E, Scheffner M, Oren M, Ciechanover A, Schwartz A. Degradation of the E7 human papillomavirus oncoprotein by the ubiquitin-proteasome system: targeting via ubiquitination of the N-terminal residue. Oncogene 2000; 19: 5944-5950.

32. Bloom J, Amador V, Bartolini F, DeMartino G, Pagano M. Proteasome-mediated degradation of p21 via N-terminal ubiquitinylation. Cell 2003; 115: 71-82.

33. Cadwell $\mathrm{K}$, Coscoy L. Ubiquitination on nonlysine residues by a viral $\mathrm{E} 3$ ubiquitin ligase. Science 2005; 309: 127-130.

34. Tokarev AA, Munguia J, Guatelli JC. Serine-threonine ubiquitination mediates downregulation of BST-2/tetherin and relief of restricted virion release by HIV-1 Vpu. $\checkmark$ Virol 2011; 85: 51-63.

35. Hao Y, Sekine K, Kawabata A, Nakamura H, Ishioka T, Ohata H et al. Apollon ubiquitinates SMAC and caspase-9, and has an essential cytoprotection function. Nat Cell Biol 2004; 6 : 849-860.

36. Bartke T, Pohl C, Pyrowolakis G, Jentsch S. Dual role of BRUCE as an antiapoptotic IAP and a chimeric E2/E3 ubiquitin ligase. Mol Cell 2004; 14: 801-811.

37. Chai J, Du C, Wu JW, Kyin S, Wang X, Shi Y. Structural and biochemical basis of apoptotic activation by Smac/DIABLO. Nature 2000; 406: 855-862.

38. Venken $\mathrm{KJ}, \mathrm{He} \mathrm{Y}$, Hoskins RA, Bellen HJ. P[acman]: a BAC transgenic platform for targeted insertion of large DNA fragments in D. melanogaster. Science 2006; 314: 1747-1751.

39. Bischof J, Maeda RK, Hediger M, Karch F, Basler K. An optimized transgenesis system for Drosophila using germ-line specific phiC31 integrases. Proc Natl Acad Sci U S A 2007; 104: $3312-3317$

40. Brand $\mathrm{AH}$, Perrimon N. Targeted gene expression as a means of altering cell fates and generating dominant phenotypes. Development 1993; 118: 401-415.

Supplementary Information accompanies the paper on Cell Death and Differentiation website (http://www.nature.com/cdd) 